\title{
Insights from mathematical modeling for convection-enhanced intraputamenal delivery of GDNF
}

\author{
Elena Belova $^{1}$ - Christopher L. Shaffer ${ }^{1}$ - Patrick E. Trapa ${ }^{1}$ \\ Received: 22 September 2016 / Accepted: 18 April 2017 / Published online: 11 May 2017 \\ (C) The Author(s) 2017. This article is an open access publication
}

\begin{abstract}
Glial cell line-derived neurotrophic factor (GDNF) is a potential therapy for Parkinson's disease (PD) promoting survival and functional recovery of dopaminergic neurons when delivered to the degenerated striatum. To study the aspects of intraputamenal delivery of GDNF, a mathematical model of recombinant methionyl human GDNF (rmetHuGDNF) convection in the human putamen has been developed. The convection-enhanced delivery infusions of $\mathrm{r}$ metHuGDNF were simulated at rates up to $5 \mu \mathrm{L} / \mathrm{min}$. The high-rate infusions ( $\geq 1 \mu \mathrm{L} / \mathrm{min}$ ) permit rapid and uniform distribution of drug with up to $75 \%$ of the distribution volume having a concentration within $5 \%$ of the infusate concentration. No relevant differences in distribution at infusion rates of 3 and $5 \mu \mathrm{L} / \mathrm{min}$ were found. The patterns of GDNF distribution were analyzed in relation to the anatomy of the posterior dorsal putamen, and a cylindrical shape was found to be preferable considering risks of target overflow. A magnetic resonance (MR) tracer Gd-DTPA (Magnevist ${ }^{\circledR}$ ) was evaluated as a surrogate in clinical studies, and the most accurate prediction of GDNF distribution was calculated immediately after infusion. The clearance of GDNF from the striatum is confirmed to be slow, with a half-life of ca. $19 \mathrm{~h}$.
\end{abstract}

Keywords Convection-enhanced delivery (CED) · Central nervous system (CNS) · Neurotrophic factor · Intraparenchymal infusion $\cdot$ Computational model

Elena Belova

elena.belova@pfizer.com

1 Worldwide Research \& Development, Pfizer Inc., 1 Portland Street, Cambridge, MA 02139, USA

\section{Introduction}

Intraparenchymal convection-enhanced delivery (CED) was first introduced in 1994 to overcome the blood-brain barrier (BBB) and to enhance drug distribution in the central nervous system [1]. The CED technique relies on convection established by a pressure gradient at the tip of the infusion catheter. The bulk flow augments diffusion and produces more widespread and uniform distribution of drug molecules in the brain. The CED infusion rate should be high enough to induce convection but low enough to be safely tolerated by patients. Drug distribution volume $\left(V_{\mathrm{d}}\right)$ is directly proportional to the infusion volume $\left(V_{\mathrm{i}}\right)$ with a ratio that is specific to the tissue type [2]. After cessation of CED, the drug continues to spread by diffusion alone until it is cleared from the tissue. One of the challenges for CED is reflux or backflow of infusate along the catheter track which can lead to poor coverage as well as possible adverse events (AEs) stemming from exposures outside of the region of interest. Loss due to reflux can reach up to $50 \%$ of the total $V_{\mathrm{d}}$ in high-volume infusions [3]. Modern CED platforms demonstrate the ability to minimize reflux while achieving large $V_{\mathrm{d}}$ in the target [4].

Glial cell line-derived neurotrophic factor (GDNF) [5] was originally isolated from a rat glioma cell line and identified as a growth factor promoting the survival of dopaminergic (DA) neurons. It has been examined as a drug candidate for the treatment of PD, which is marked by progressive loss of nigrostriatal DA neurons. Studies with rodent and nonhuman primate models of PD have confirmed the neurorestorative and neuroprotective effects of GDNF when delivered into the cerebral ventricles or directly into the striatum or substantia nigra [6-9]. Preclinical findings led to several trials in PD patients. In the first study, recombinant methionyl human GDNF (r-metHuGDNF) was administered intracerebroventricularly (ICV) to patients by monthly bolus 
injections. The study failed to provide clinical benefits but demonstrated significant AEs [10]. A postmortem examination of one patient revealed that the GDNF ICV treatment did not induce regeneration of nigrostriatal neurons in this patient [11]. To achieve efficacy and reduce AEs, three subsequent trials delivered r-metHuGDNF directly into the putamen of PD patients via continuous infusion driven by intraabdominal pumps. Two small open-label studies reported substantial improvements in patient motor function with adequate safety and tolerability after 6-12 months of infusion $[12,13]$. However, a third randomized double-blind placebo-controlled trial in patients with moderate-to-severe PD did not detect a significant improvement in motor outcome compared with placebo despite a modest local increase in $\left[{ }^{18} \mathrm{~F}\right] \mathrm{DOPA}$ uptake as shown by positron emission tomography (PET) [14]. Retrospectively, the limited distribution of r-metHuGDNF caused by technical challenges was hypothesized to be partly responsible for trial failure [15]. Since patient $V_{\mathrm{d}}$ were not assessed throughout the trial, the clinical performance of the infusion system was not evaluated.

More recently, a randomized double-blind placebo-controlled trial was designed to explore the benefits of a chronic intermittent dosing regimen for intraputamenal (IPu) delivery $[16,17]$. Consistent with the expectation that this regimen would increase coverage of the striatum and prevent offtarget distribution [18], the study required a distinct minimum coverage of a predefined putamenal volume of interest for patients to proceed to randomization [17]. Intermittent administration was supported by observations in rats of prolonged neurorestorative effects of GDNF in the striatum after a single intrastriatal infusion $[19,20]$. The aim of the present work was to model prospectively the distribution of r-metHuGDNF in the putamen following a short-term IPu infusion.

Our model was built on the framework introduced by Morrison et al. [21] in 1994 to mathematically describe transport of macromolecules in intraparenchymal CED. The authors used a rigid-pore assumption to model brain tissue and applied the convection-diffusion-reaction differential mass balance equation to define macromolecular transport. Their theory was broadly adopted for CED modeling, where computational results are usually validated in preclinical models [21-23]. The final structure of the model depends on the CED application, namely a microstructure of the target, properties of the drug, parameters of the CED platform, and delivery protocol.

\section{Methods}

\subsection{Mathematical model}

The modeling approach is illustrated in Fig. 1. The general convection-diffusion-reaction differential mass balance

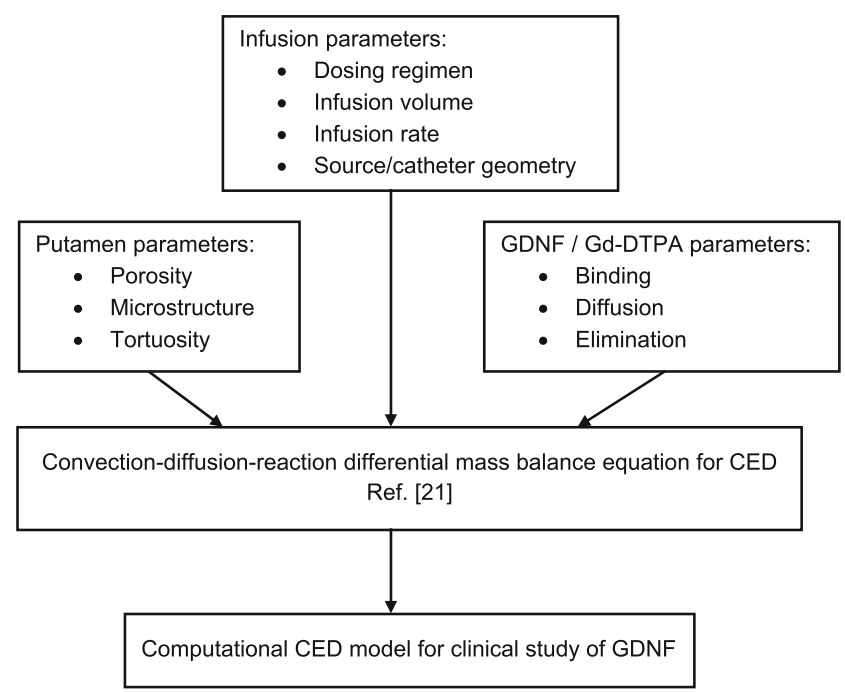

Fig. 1 Schematic of the generation of the applied model from CED theory

equation for CED was defined in Morrison et al. [21]. Applying it to the transport of $r$-metHuGDNF in the putamen, we neglected macromolecular binding, both specific and nonspecific, in interstitial fluid (ISF). Our assumption was supported by studies in non-human primates (NHPs), where it was found that in IPu CED the difference in $V_{\mathrm{d}}$ between GDNF and the MRI tracer gadoteridol (Gd) was slight [24], suggesting the negligible role of binding in the distribution process. The next assumption was to ignore the loss of GDNF across the capillary walls because of the low ISF flow rate. As it will be shown in Section 3, in the present model, the infusion times necessary to simulate $V_{\mathrm{d}}$ were about $1-2 \mathrm{~h}$ depending on the CED rate, while an ISF turnover time is

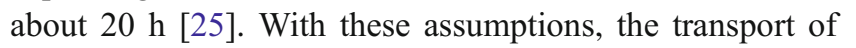
non-binding macromolecules is governed by the equation

$\phi \frac{\partial C_{\mathrm{ISF}}}{\partial t}=\nabla \cdot\left(\phi \mathbf{D}_{\mathbf{t}} \cdot \nabla C_{\mathrm{ISF}}\right)-\nabla \cdot\left(\phi \vec{v}_{\mathrm{ISF}} C_{\mathrm{ISF}}\right)-k_{\mathrm{irr}} C_{\mathrm{ISF}}$,

where $C_{\mathrm{ISF}}$ is the concentration of r-metHuGDNF or GdDTPA in ISF, $\phi$ is the tissue porosity, $\vec{v}_{\text {ISF }}$ is the interstitial velocity; $\mathbf{D}_{\mathbf{t}}$ is the macromolecular diffusion tensor in the putamen, and $k_{\text {irr }}$ is a first-order degradation rate constant $[2,21]$.

The diffusion tensor is defined by the regional microstructure of the target. The fractional anisotropy (FA) of the putamen was measured in diffusion tensor imaging (DTI) studies in healthy elderly subjects and patients with mild-to-moderate PD [26]. In almost all cases, the reported FA was below an approximate threshold value for the gray matter $(\mathrm{FA}<0.275)$ defined by Kim et al. [22]. The latter means an isotropic distribution of macromolecules within the putamen with a constant value for diffusivity [27]:

$D_{t}=\frac{D}{\lambda^{2}}$, 
where $D$ is the diffusion coefficient in the free medium and $\lambda$ is the tortuosity characterizing the hindrance to diffusion in brain ISF.

To define the interstitial fluid velocity $\vec{v}_{\text {ISF }}$, we solved the equation for continuity of brain ISF:

$\nabla \cdot \vec{v}_{\mathrm{ISF}}=0$

where 0 in the right part of the equation is consistent with our assumption to ignore ISF exchange in the simulations. Assuming a spherical symmetry of distribution from the catheter in the isotropic target and given the boundary condition at the catheter tip [28],

$Q=4 \pi r_{0}^{2} \phi v_{r}$,

where $Q$ is the volumetric infusion rate and $r_{0}$ is the radius of the catheter tip; the solution for the radial interstitial velocity is

$v_{r}=\frac{Q}{4 \pi \phi r^{2}}$,

where $r$ is the distance from the catheter tip, $r>r_{0}$.

Thus, the final model equation defining the transport of either non-binding r-metHuGDNF or Gd-DTPA in the putamen is

$\frac{\partial C_{\mathrm{ISF}}}{\partial t}=\frac{D}{\lambda^{2}} \frac{1}{r^{2}} \frac{\partial}{\partial r}\left(r^{2} \frac{\partial C_{\mathrm{ISF}}}{\partial r}\right)-v_{r} \frac{\partial C_{\mathrm{ISF}}}{\partial r}-k C_{\mathrm{ISF}}$,

where $k$ represents a first-order elimination rate constant assumption and was calculated from the preclinical studies for recombinant human GDNF [19]. Equation (6) was solved numerically in MATLAB R2010b (MathWorks, Natick, MA) using a finite element technique. For convenience, Eq. (6) was solved for dimensionless concentration $C_{\text {norm }}$ via scaling the equation to the initial infusate concentration $C_{0}$ :

$C_{\text {norm }}=\frac{C_{\mathrm{ISF}}}{C_{0}}$

\subsection{Elimination rate}

Two approaches were used to determine the rate of elimination $k$ in Eq. (6). First, the clearance rate was calculated from the published pharmacokinetic (PK) study of a single GDNF infusion into the rat striatum [19]. In that study, two doses of recombinant human GDNF (15 and $3 \mu \mathrm{g}$ ) were infused by CED, and GDNF concentration in the whole striatum was measured at $3,7,14,21$, and 28 days post-infusion. These data were fit with a two-compartment PK model in NONMEM v.7.2 (ICON Development Solutions, Hanover, $\mathrm{MD})$, where the brain extracellular space was taken as the central compartment to estimate the rate constants, mean (SE): $k_{10}=3.72(0.44) \times 10^{-2} h^{-1}, k_{12}=$ $7.12(1.20) \times 10^{-4} h^{-1}$, and $k_{21}=3.81(0.76) \times 10^{-3} h^{-1}$. Since $k_{12}$ $<<k_{10}$, then $k_{e l} \sim k_{10}$, a first-order elimination is able to fit the GDNF concentration at 3 and 7 days after infusion, while the second exponent input $\left(k_{21}\right)$ becomes important at later time points. First-order elimination was therefore deemed sufficient to capture initial distribution, and the rate constant $k=k_{10}$ was introduced into the model. The calculated half-life of GDNF in the striatum was approximately $19 \mathrm{~h}$.

The elimination rate was also calculated using the ISF flow rate explicitly assuming that clearance by ISF flux is the main elimination pathway. If the ISF outflow rate is approximately $0.17 \mu \mathrm{L}$ per gram of brain per minute [25], then for a typical rat brain weighing $1.8 \mathrm{~g}$, the flow rate $Q_{\mathrm{ISF}}$ is $0.306 \mu \mathrm{L} / \mathrm{min}$. The total ISF volume, $540 \mu \mathrm{L}$, was projected from the assumption of brain tissue porosity:

$\phi=\frac{V_{\mathrm{ISF}}}{V_{\text {brain }}}$,

where the $\phi$ value is between 0.2 and 0.4 [21]. Therefore, $\phi=$ 0.3 was used in the present work. Consequently, the elimination rate by ISF flux is

$k=\frac{Q_{\mathrm{ISF}}}{V_{\mathrm{ISF}}}$,

where $k=3.40 \cdot 10^{-2} h^{-1}$. This rate was very close to the prediction from the rat PK study suggesting that the main mechanism of elimination of GDNF from the striatum is clearance by the slow ISF flow.

\subsection{Diffusion parameters}

Diffusion parameters of r-metHuGDNF, a homodimer with a molecular weight of $30.4 \mathrm{kDa}$ [20], were extrapolated from the diffusion study of a bioactive rhodamine nerve growth factor (NGF) conjugate $(26.5 \mathrm{kDa})$ in the rat striatum [29, 30] using the Stokes-Einstein relation and a standard assumption of a spherical shape for the diffusing molecules: $D_{\mathrm{GDNF}}=$ $1.3 \cdot 10^{-6} \mathrm{~cm}^{2} / \mathrm{s} ; \lambda_{\mathrm{GDNF}}=2.2$. The diffusion coefficient of $\mathrm{Gd}-$ DTPA (Magnevist ${ }^{\circledR}, 938 \mathrm{Da}$ ) was extrapolated from the obtained $D_{\mathrm{GDNF}}$, explicitly assuming an inverse one-third power dependence of diffusivity on molecular weight: $D_{\mathrm{Gd}-\mathrm{DTPA}}=$ $4.1 \cdot 10^{-6} \mathrm{~cm}^{2} / \mathrm{s}$. The Gd-DTPA tortuosity value, $\lambda_{\mathrm{Gd}-\mathrm{DTPA}}=$ 1.6 , was chosen from the range of 1.5-1.6 measured for small molecules in brain ISF [30].

\subsection{Loss during infusion}

To estimate the amount of drug cleared in the process of infusion, the following assumptions were made: (1) intraputamenal drug concentration remains unchanged during 
the short infusion and equals the infusate concentration, $C_{0}$; (2) $V_{\mathrm{d}}$ is proportional to $V_{\mathrm{i}}$ with a constant ratio $\left(V_{\mathrm{d}} / V_{\mathrm{i}}\right)$; (3) infusion occurs at a constant rate $Q$; (4) distribution caused by diffusion is negligible in CED; and (5) binding, both specific and non-specific, was ignored. Based on these assumptions, the differential mass balance equations are

$\frac{d A(t)}{d t}=-k A(t)+Q C_{0}\left(\frac{V_{\mathrm{d}}}{V_{\mathrm{i}}}\right)$,

$\frac{d A_{\text {Loss }}(t)}{d t}=k A(t)$,

where $A$ is the total amount of drug while $A_{\text {Loss }}$ is the amount cleared (i.e., lost), and initial conditions are

$A(0)=0$,

$A_{\text {Loss }}(0)=0$.

The equations were solved symbolically in MATLAB R2010b (MathWorks, Natick, MA), and the following solution for $A_{\text {Loss }}$ was obtained:

$A_{\text {Loss }}(t)=Q C_{0}\left(\frac{V_{\mathrm{d}}}{V_{\mathrm{i}}}\right)\left(t-\frac{1-e^{-k t}}{k}\right)$.

\subsection{Distribution in target}

In previous clinical trials, the target for CED infusions was the posterior dorsal putamen since this is the region most depleted of dopamine in PD [14]. We have projected $V_{\mathrm{d}}$ in this target using the measurements manually extracted from the ICBM (International Consortium for Brain Mapping) template atlas using Amira v.5.5 (FEI Visualization Sciences Group, Bordeaux, France) for the left posterior dorsal putamen: $1.92 \mathrm{~cm}$ (length), $1.15 \mathrm{~cm}$ (height), and 1.12 or $0.90 \mathrm{~cm}$ (width, the measurement depends on the location as shown in Fig. 2a). Reported MRI-based measurements suggest the putamen volume in PD patients is $3.98 \pm 0.15 \mathrm{~cm}^{3}$ [31]. We assumed that the target is roughly $25 \%$ of total putamen and predicted a volume of interest of $1 \mathrm{~mL}$ per putamen. Since the employed CED system is proposed to utilize four microcatheters per patient [16], or two catheters per putamen, our estimation for $V_{d}$ per catheter was $0.5 \mathrm{~mL}$.

In isotropic tissues, a reflux-resistant CED catheter produces a uniform spherical $V_{\mathrm{d}}$ around the infusion site (Fig. 2b):

$V_{\mathrm{d}}=\frac{4}{3} \pi r^{3}$,

where $r$ is the distributional radius. Thus, for $0.5 \mathrm{~mL} V_{\mathrm{d}}$, the corresponding distributional diameter is $0.98 \mathrm{~cm}$ (Table 1). In the model, the volume occupied by the catheter tip in the target was negligible compared to the total achieved $V_{\mathrm{d}}$. For

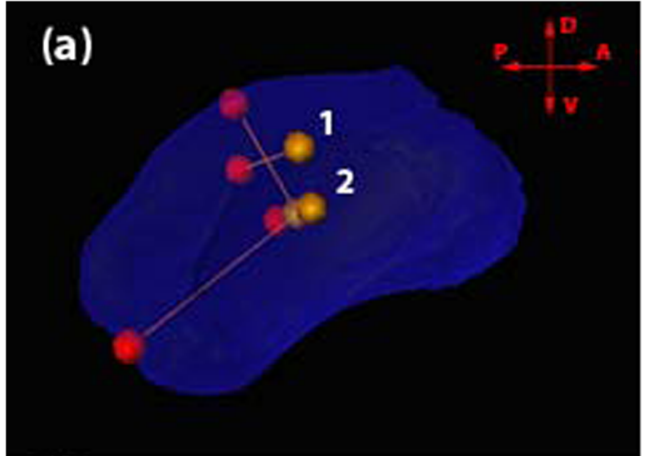

(b)

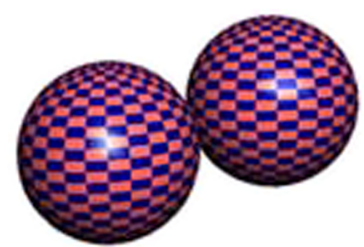

(c)

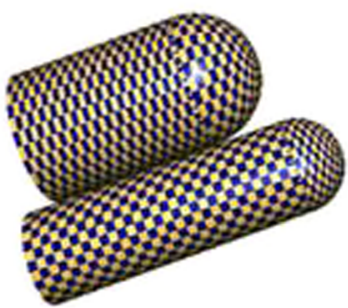

Fig. 2 The left human putamen from the ICBM atlas with the outlined region of interest (ROI). Labels 1 and 2 indicate two measurements of ROI width. Orientation is indicated by arrows: $P$ posterior, $D$ dorsal, $A$ anterior, $V$ ventral (a). Distribution patterns for two catheters with isolated either spherical $V_{\mathrm{d}}(\mathbf{b})$ or cylindrical $V_{\mathrm{d}}(\mathbf{c})$

example, a typical cannula with a length of $1 \mathrm{~cm}$ and an outer diameter of $1 \mathrm{~mm}$ [3] occupies a volume of about $8 \mu \mathrm{L}$.

Catheter design impacts the shape of the distribution. Cylinder-like $V_{\mathrm{d}}$ are more typical for conventional catheters because of reflux (i.e., backflow) [3]. When reflux is controlled and constrained within the target, the cylindrical distribution may be more suitable for non-spherical regions of interest. In this case, the total $V_{\mathrm{d}}$ can be estimated as a sum of two volumes, the hemisphere formed below the tip and the cylindrical volume refluxed above the tip (Fig. 2c):

$V_{\mathrm{d}}=\frac{2}{3} \pi r^{3}+\pi r^{2} h$,

where $h$ is the reflux length and $(r+h)$ is the total length. The total length and diameter are related inversely. For example, for projected $0.5 \mathrm{~mL} V_{\mathrm{d}}$, two possible scenarios were calculated: $0.70 \mathrm{~cm}$ distributional length and $1.12 \mathrm{~cm}$ distributional diameter, or $1.90 \mathrm{~cm}$ distributional length and $0.60 \mathrm{~cm}$ diameter (Table 1). Also, in the case of cylindrical $V_{\mathrm{d}}$, the angle of catheter inclination becomes an additional parameter to optimize the volume. 
Table 1 Anatomical parameters of the human posterior dorsal putamen and models of $0.5 \mathrm{~mL} V_{\mathrm{d}}$

\begin{tabular}{|c|c|c|c|}
\hline Dimensions & ICBM measurements $^{\mathrm{a}}(\mathrm{cm})$ & Spherical $V_{\mathrm{d}} \operatorname{model}^{\mathrm{b}}(\mathrm{cm})$ & Cylindrical $V_{\mathrm{d}} \operatorname{model}^{\mathrm{b}}(\mathrm{cm})$ \\
\hline Length & 1.92 & 0.98 & $0.70-1.90$ \\
\hline Height & 1.15 & 0.98 & $0.60-1.12$ \\
\hline Width & $1.12,0.90^{\mathrm{c}}$ & 0.98 & $0.60-1.12$ \\
\hline
\end{tabular}

${ }^{\text {a }}$ Measurements made for the left posterior dorsal putamen

${ }^{\mathrm{b}}$ Calculations per catheter; each putamen contains two catheters

${ }^{\mathrm{c}}$ Measured at two levels, labels 1 and 2 from Fig. 2a, respectively

\section{Results}

\subsection{GDNF distribution at different infusion rates}

Infusions were modeled at several possible rates. Simulation parameters are listed in Table 2. In the early GDNF PD trials, $\mathrm{IPu}$ infusions were continuous at a non-CED rate of $0.1 \mu \mathrm{L} /$ $\min [12]$. However, the minimal rate to induce convection is $0.5 \mu \mathrm{L} / \mathrm{min}$ [18]. A novel improved CED system can deliver reflux-free infusions at rates of up to $5 \mu \mathrm{L} / \mathrm{min}$ [3]. Safety of CED infusions has been assessed in preclinical and clinical studies to a reported rate limit of $10 \mu \mathrm{L} / \mathrm{min}$ [2]. Based on this, the following infusion rates were investigated: $0.1,1,3$, and $5 \mu \mathrm{L} / \mathrm{min}$.

Concentration profiles have been simulated for all four rates (Fig. 3). Infusions at the highest rates, 3 and $5 \mu \mathrm{L} / \mathrm{min}$, produce nearly identical concentration profiles. Considering the geometry of the target and estimations for $V_{\mathrm{d}}$ made beforehand (Table 1), the distribution of GDNF is expected within radial distances $(r)$ of 0.30 to $0.56 \mathrm{~cm}$ from the catheter. Infusions were simulated from the sphere around the catheter tip at a radius of $0.2 \mathrm{~cm}\left(r_{0}^{\prime}\right)$ to avoid numerical instability caused by very high instantaneous exit velocity $v_{r}$ near the catheter tip (e.g., with a typical diameter of $0.06 \mathrm{~cm}$ ) [3]. A $V_{\mathrm{i}}$ of $0.105 \mathrm{~mL}$ was chosen for simulation to reach $C_{\text {norm }}=$ 0.5 at a distance of ca. $0.55 \mathrm{~cm}$, although the profile for the lowest infusion rate of $0.1 \mu \mathrm{L} / \mathrm{min}$ broadened due to diffusion

Table 2 Simulation parameters

\begin{tabular}{lll}
\hline Parameter & Symbol & Value \\
\hline Porosity & $\phi$ & 0.3 \\
Distribution volume $(\mathrm{mL})$ & $V_{\mathrm{d}}$ & 0.5 \\
Distribution distance $(\mathrm{cm})$ & $r$ & $0.3-0.56$ \\
Source radius $(\mathrm{cm})$ & $r_{0}$ & 0.2 \\
Infusion rate $(\mu \mathrm{L} / \mathrm{min})$ & $Q$ & $0.1-5$ \\
Infusion volume $(\mathrm{mL})$ & $V_{\mathrm{i}}$ & 0.105 \\
Diffusion coefficient $\left(\mathrm{cm}^{2} / \mathrm{s}\right)$ & $D_{\mathrm{GDNF}}$ & $1.3 \times 10^{-6}$ \\
& $D_{\mathrm{Gd}-\mathrm{DTPA}}$ & $4.1 \times 10^{-6}$ \\
Tortuosity & $\lambda_{\mathrm{GDNF}}$ & 2.2 \\
& $\lambda_{\mathrm{Gd}-\mathrm{DTPA}}$ & 1.6 \\
Elimination rate constant $\left(\mathrm{h}^{-1}\right)$ & $k$ & $3.72 \times 10^{-2}$ \\
\hline
\end{tabular}

and $C_{\text {norm }}=0.5$ was shifted to the left after the infusion (Table 3).

The Peclet number $(\mathrm{Pe})$, which describes the ratio of mass transfer by convection to diffusion, was used to characterize the GDNF distribution at each infusion rate. Within the model, this ratio equals

$$
P e=\frac{Q}{4 \pi \phi \frac{D_{\mathrm{GDNF}}}{\lambda_{\mathrm{GDNF}}^{2}} r} .
$$

$P e$ is decreased proportionally with the radial distance from the catheter $r$. The minimal $P e$ was calculated for each infusion rate at the distance associated with $C_{\text {norm }}=0.5$.

To determine the homogeneity of distribution, a ratio of high-concentration coverage to the total $V_{\mathrm{d}}$ was calculated. The threshold values for the high-concentration $V_{\mathrm{d}}$ and total $V_{\mathrm{d}}$ were $C_{\text {norm }}=0.95$ and $C_{\text {norm }}=0.05$, respectively (Table 3 ). For the 1,3 , and $5 \mu \mathrm{L} / \mathrm{min}$ infusion rates, the highconcentration portion accounts for 45,67 , and $75 \%$ of total $V_{\mathrm{d}}$, respectively. Such uniform distribution occurs because bulk flow dominates over diffusion as characterized by $P e>>1$. At $0.1 \mu \mathrm{L} / \mathrm{min}$, the Peclet number is close to 1 and both processes, bulk flow and diffusion, impact the distribution. Diffusion not only broadens the coverage but also diminishes homogeneity, reducing the high-concentration portion to $5 \%$.

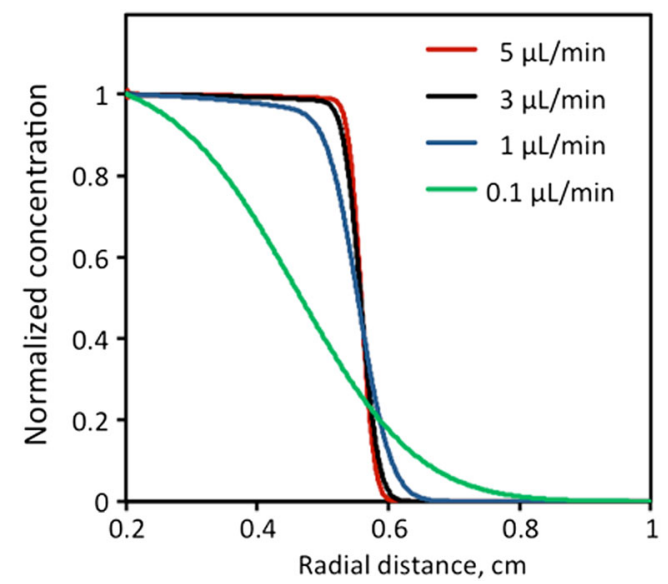

Fig. 3 R-metHuGDNF concentration profiles developed at different infusion rates. All infusions were simulated from the $0.2-\mathrm{cm}$ sphere and lasted until the total $V_{\mathrm{i}}$ of $0.105 \mathrm{~mL}$ was delivered 
Table 3 R-metHuGDNF distribution parameters at different infusion rates

\begin{tabular}{lllllll}
\hline Rate $(\mu \mathrm{L} / \mathrm{min})$ & \multicolumn{2}{c}{$C_{\text {norm }}=0.5$} & & $V_{\mathrm{d}}(\mathrm{mL})$ & \multicolumn{3}{c}{$V_{\mathrm{d}}^{0.95} / V_{\mathrm{d}}^{0.05}$} \\
\cline { 2 - 3 } & $r(\mathrm{~cm})$ & $P e$ & & $C_{\text {norm }} \leq 0.95$ & $C_{\text {norm }} \leq 0.05$ & \\
\hline 0.1 & 0.467 & 3.5 & 0.072 & 1.479 & 0.05 \\
1 & 0.553 & 30 & 0.444 & 0.997 & 0.45 \\
3 & 0.558 & 88 & 0.590 & 0.877 & 0.67 \\
5 & 0.558 & 147 & 0.630 & 0.837 & 0.75 \\
\hline
\end{tabular}

The calculated $V_{\mathrm{d}}$ per catheter in this study is $0.5 \mathrm{~mL}$. Assuming the linear dependence between the infusion and distribution volumes and a $V_{\mathrm{d}} / V_{\mathrm{i}}=3.87$ measured in the NHP putamen [24], the required infusion volume is projected as $0.13 \mathrm{~mL}$ per catheter. In the model, all high-rate infusions $(\geq 1 \mu \mathrm{L} / \mathrm{min}$ ) resulted in a uniform square-shaped concentration profile, a defining characteristic of convection-dominated transport. To deliver the projected $V_{\mathrm{i}}$, the infusion time is set as 130,43 , and $26 \mathrm{~min}$ for 1,3 , and $5 \mu \mathrm{L} / \mathrm{min}$ rates, respectively. Although the elimination of GDNF from the target is slow, some amount of the infusate is cleared over the course of the infusion. Assuming a constant concentration of infusate in the target $\left(C_{\text {norm }}=1\right)$, we have estimated the portion of $V_{\mathrm{d}}$ which is cleared during the infusion using Eq. (12). Calculated $V_{\text {Loss }}$ were $0.020,0.007$, and $0.004 \mathrm{~mL}$ for infusions at 1,3 , and $5 \mu \mathrm{L} / \mathrm{min}$, respectively. Greater loss occurs when the drug is infused at slower rates because the infusion time is longer. This effect was also observed in the simulations, when the $1 \mu \mathrm{L} / \mathrm{min}$ concentration profile, while still steep, had a slightly lower amplitude $\left(C_{\text {norm }}\right)$ than the other two profiles. No differences were found between the 3 and $5 \mu \mathrm{L} / \mathrm{min}$ rates; they produced nearly identical concentration profiles characterized by approximately $1 \%$ of $V_{\mathrm{d}}$ loss during the infusion phase.

\subsection{Correlation between Gd-DTPA and GDNF distributions}

The distribution of drug delivered via CED can be approximated by imaging test infusions of MRI-visible tracers, such as clinical-grade Gd-DTPA (Magnevist ${ }^{\circledR}$ ) [32]; however, the transport properties of small and large molecules, in this case Gd-DTPA $(<1 \mathrm{kDa})$ and r-metHuGDNF (ca. $30 \mathrm{kDa})$, differ. Simulations were performed to compare the distributions using an infusion rate of $5 \mu \mathrm{L} / \mathrm{min}$ (Fig. 4). Both infusions were again simulated from the sphere around the catheter with a radius of $0.2 \mathrm{~cm}\left(r_{0}^{\prime}\right)$ and a $V_{\mathrm{i}}$ of $0.105 \mathrm{~mL}$. Simulated concentration profiles were plotted immediately postinfusion and $2 \mathrm{~h}$ thereafter to illustrate the temporal dependence of Gd-DTPA and r-metHuGDNF distributions. Our previously described estimations of the GDNF elimination rate suggest that the main GDNF clearance mechanism appears to

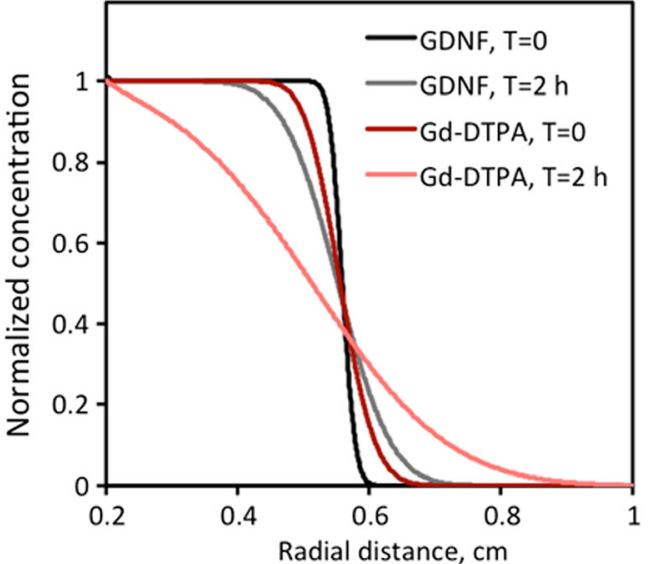

Fig. $45 \mu \mathrm{L} / \mathrm{min}$ infusion concentration profiles of r-metHuGDNF (black lines) and Gd-DTPA (red lines) immediately ( $T=0$, colorful lines) and $2 \mathrm{~h}$ after $(T=2 \mathrm{~h}$, pale lines $)$

be ISF bulk flow; therefore, the elimination rate should not depend on the size of impermeable molecules. Thus, the elimination rate of Gd-DTPA would be expected to equal that of GDNF since neither cross the intact BBB. Consequently, the clearance process should not significantly affect the difference in distribution between the two compounds within the 2-h time frame, and hence, it was ignored in simulations.

To compare the Gd-DTPA and GDNF distributions, $V_{\mathrm{d}}$ for both molecules were calculated from the simulation using Eq. (13) and assuming a uniform spherical distribution in the target. The distributional radii for each $V_{\mathrm{d}}$ were defined at the tissue concentration threshold value, arbitrarily chosen as a $10 \%$ of $C_{0}\left(C_{\text {norm }}=0.1\right)$. Simulated $V_{\mathrm{d}}$ of GDNF were $0.81 \mathrm{~mL}$ immediately after the infusion was ended $(T=0)$ and $1.07 \mathrm{~mL} 2$ later $(T=2 \mathrm{~h}) . V_{\mathrm{d}}$ of Gd-DTPA were $0.95 \mathrm{~mL}$ immediately after the infusion $(T=0)$ and $1.58 \mathrm{~mL} 2 \mathrm{~h}$ after the infusion (Table 4). Thus, within $2 \mathrm{~h}$, the $V_{d}$ increase was 1.3-fold for GDNF and 1.7-fold for Gd-DTPA. The correlation between Gd-DTPA and GDNF $V_{\mathrm{d}}$ was calculated as a $V_{\mathrm{d}}^{\mathrm{Gd}-\mathrm{DTPA}} / V_{\mathrm{d}}^{\mathrm{GDNF}}$ ratio and found to be 1.2 immediately after the infusion $(T=0)$ and $1.52 \mathrm{~h}$ later $(T=2 \mathrm{~h})$.

The diffusion of molecules depends on their size with smaller molecules traveling faster and therefore farther in the tissue. The tortuosity values characterizing the hindrance to diffusion in brain ISF are also reduced for small molecules [30]. As a result, an effective diffusion coefficient of $\mathrm{Gd}-$

Table 4 Correlation between r-metHuGDNF and Gd-DTPA distribution volumes $\left(V_{\mathrm{d}}\right)$ immediately and $2 \mathrm{~h}$ after ending a $5 \mu \mathrm{L} / \mathrm{min}$ infusion

\begin{tabular}{lll}
\hline & $T=0$ & $T=2 \mathrm{~h}$ \\
\hline$V_{\mathrm{d}}^{\text {Gd -DTPA }}(\mathrm{mL})$ & 0.95 & 1.58 \\
$V_{\mathrm{d}}^{\text {GDNF }}(\mathrm{mL})$ & 0.81 & 1.07 \\
$V_{\mathrm{d}}^{\text {Gd }- \text { DTPA }} / V_{\mathrm{d}}^{\text {GDNF }}$ & 1.2 & 1.5 \\
\hline
\end{tabular}


DTPA in the striatum, $D_{t, \text { Gd - DTPA }}$, defined in Eq. (2), was 6fold greater than $D_{t}$, GDNF ; therefore, larger $V_{\mathrm{d}}$ were found in Gd-DTPA simulations. The more accurate prediction of $r$ metHuGDNF $V_{\mathrm{d}}$ was found during the infusion process, when the difference in diffusion was compensated by bulk flow.

\section{Discussion}

Modeling suggests that short, intermittent, CED infusions afford the opportunity to optimize both the volume and the pattern of GDNF distribution. Such precise delivery is particularly beneficial considering the potential risks associated with exposing non-target structures to GDNF [18]. Recently, distribution shape has been tailored through modifications of the step length of a recessed-step catheter [4]. The present analysis, based on the anatomy of the human putamen, suggests that cylindrical patterns permit more flexibility in target coverage. Specifically, cylindrical distribution with custom height and width may be used to accommodate intersubject anatomical variability of target structures.

The present model is a preliminary approach to describe an $\mathrm{IPu}$ delivery of GDNF in patients. Further refinement requires clinical data. For example, the next step will be incorporating DTI data to project the degree of anisotropy and inhomogeneity of the putamen and surrounding tissue. The MR-DTI technique measures the effective tensor of water diffusion in the tissue which is sensitive to the tissue microstructure. The water diffusion tensor is then used to assign directionality to $\mathbf{D}_{\mathbf{t}}$ tensor in the model. The methodology of DTI-based tensor calibration and tissue segmentation to build a 3D computational model for CED has been reported in detail elsewhere $[22,23,33]$. The studies show that within the gray matter, diffusivity and hydraulic conductivity are nearly identical in all directions [2, 22]. Indeed, the assumption of an isotropic target based on published DTI data has been used in the present model, and $\mathbf{D}_{\mathbf{t}}$ was approximated by a constant value. On the other hand, the studies show that in the white matter, the bulk flow is preferential along the fiber tracts $[2,23]$; consequently, the tensors should project the anisotropy of the target in the model. In the case of complex structures, such as the striatum, a voxel-by-voxel segmentation of target and surroundings into either the gray or white matter should provide more accurate predictions for $V_{\mathrm{d}}$. The intrinsic tissue transport properties may also be altered because of edema and tissue deformation caused by CED. In the present model, these effects were accounted for using an increased value of porosity $\phi$, while in normal tissue, $\phi=0.2$ [21]. We expect to account for anisotropy of these effects in the 3D model as well.

The numerical limitation of the present model is an inability to simulate the distribution within the catheter surrounding due to the high calculated velocity $v_{r}$ immediately after the fluid exits the catheter. The missing volume was calculated as
$0.03 \mathrm{~mL}$. Since the model was applied to study the clinical application of CED with a projected $V_{\mathrm{d}}$ of $0.5 \mathrm{~mL}$, this numerical limitation was not meaningful. Meanwhile, examples of CED models for small-volume simulations (e.g., in rodents $[22,23])$ demonstrate that this limitation can be overcome with a computational fluid dynamic software approach.

In conclusion, several aspects of IPu delivery of $\mathrm{r}$ metHuGDNF have been studied by means of modeling. The results confirm the slow elimination of GDNF from the striatum with a half-life of about $19 \mathrm{~h}$ and suggest that local ISF flow is the primary clearance mechanism. The modeling predicts that a cylindrical pattern of distribution is more favorable for the posterior dorsal putamen, increasing coverage and reducing the risk of distribution outside of the region of interest. No significant differences in distribution were found between infusion rates of 3 and $5 \mu \mathrm{L} / \mathrm{min}$; both CED rates result in rapid and efficient r-metHuGDNF distribution. Finally, because of its smaller molecular weight, and therefore its greater effective diffusion, clinical-grade Gd-DTPA (Magnevist ${ }^{\circledR}$ ) overpredicts the distribution volume of GDNF especially when imaging is performed post-infusion.

Acknowledgements We would like to thank our colleagues Dr. Kevin Hallock and Dr. Patrick Tierney for assistance with the ICBM atlas and human putamen measurements. The authors are full-time employees of Pfizer Inc. and own and/or hold options and/or restricted stock units for the company's publicly traded shares.

Open Access This article is distributed under the terms of the Creative Commons Attribution 4.0 International License (http:// creativecommons.org/licenses/by/4.0/), which permits unrestricted use, distribution, and reproduction in any medium, provided you give appropriate credit to the original author(s) and the source, provide a link to the Creative Commons license, and indicate if changes were made.

\section{References}

1. Bobo RH, Laske DW, Akbasak A, Morrison PF, Dedrick RL, Oldfield EH (1994) Convection-enhanced delivery of macromolecules in the brain. Proc Natl Acad Sci U S A 91:2076-2080

2. Lonser RR, Sarntinoranont M, Morrison PF, Oldfield EH (2015) Convection-enhanced delivery to the central nervous system. J Neurosurg 122:697-706

3. Gill T, Barua NU, Woolley M, Bienemann AS, Johnson DE, O'Sullivan S, Murray G, Fennely C, Lewis O, Irving C, Wyatt MJ, Moore P, Gill SS (2013) In vitro and in vivo testing of a novel recessed-step catheter for reflux-free convection-enhanced drug delivery to the brain. J Neurosci Methods 219:1-9

4. Lewis O, Woolley M, Johnson D, Rosser A, Barua NU, Bienemann AS, Gill SS, Evans S (2016) Chronic, intermittent convectionenhanced delivery devices. J Neurosci Methods 259:47-56

5. Lin L-FH, Doherty DH, Lile JD, Bektesh S, Collins F (1993) GDNF: a glial cell line-derived neurotrophic factor for midbrain dopaminergic neurons. Science 260:1130-1132

6. Tomac A, Lindqvist E, Lin L-FH, Ogren SO, Young D, Hoffer BJ, Olson L (1995) Protection and repair of the nigrostriatal dopaminergic system by GDNF in vivo. Nature 373:335-339

7. Gash DM, Zhang Z, Ovadia A, Cass WA, Yi A, Simmerman L, Russell D, Martin D, Lapchack PA, Collins F, Hoffer BJ, Gerhardt 
GA (1996) Functional recovery in parkinsonian monkeys treated with GDNF. Nature 380:252-255

8. Bjorklund A, Rosenblad C, Winkler C, Kirik D (1997) Studies on neuroprotective and regenerative effects of GDNF in a partial lesion model of Parkinson's disease. Neurobiol Dis 4:186-200

9. Grondid R, Zhang Z, Yi A, Cass WA, Maswood N, Andersen AH, Elsberry DD, Klein MC, Gerhardt GA, Gash DM (2002) Chronic, controlled GDNF infusion promotes structural and functional recovery in advanced parkinsonian monkeys. Brain 125:2191-2201

10. Nutt JG, Burchiel KJ, Comella CL, Jankovic J, Lang AE, Laws ER, Lozano AM, Penn RD, Simpson RK, Stacy M, Wooten GF (2003) Randomized, double-blind trial of glial cell line-derived neurotrophic factor (GDNF) in PD. Neurology 60:69-73

11. Kordower JH, Palfi S, Chen E-Y, Ma SY, Sendera T, Cochran EJ, Mufson EJ, Penn R, Goetz CG, Comella CD (1999) Clinicopathological findings following intraventricular glialderived neurotrophic factor treatment in a patient with Parkinson's disease. Ann Neurol 46:419-424

12. Gill SS, Patel NK, Hotton GR, O'Sullivan K, McCarter R, Bunnage M, Brooks DJ, Svendsen CN, Heywood P (2003) Direct brain infusion of glial cell line-derived neurotrophic factor in Parkinson disease. Nat Med 9(5):589-595

13. Slevin JT, Gerhardt GA, Smith CD, Gash DM, Kryscio R, Young B (2005) Improvement of bilateral motor functions in patients with Parkinson disease through the unilateral intraputamenal infusion of glial cell line-derived neurotrophic factor. J Neurosurg 102(2):216-222

14. Lang AE, Gill S, Patel NK, Lozano A, Nutt JG, Penn R, Brooks DJ, Hotton G, Moro E, Heywood P, Brodsky MA, Burchiel K, Kelly P, Dalvi A, Scott B, Stacy M, Turner D, Wooten VGF, Elias WJ, Laws ER, Dhawan V, Stoessl AJ, Matcham J, Coffey RJ, Traub M (2006) Randomized controlled trial of intraputamenal glial cell line-derived neurotrophic factor infusion in Parkinson disease. Ann Neurol 59:459-466

15. Salvatore MF, Ai Y, Fischer B, Zhang AM, Grondin RC, Zhang Z, Gerhardt GA, Gash DM (2006) Point source concentration of GDNF may explain failure of phase II clinical trial. Exp Neurol 202:497-505

16. Gill SS, White E, Barua N, Bienemann A, Patel N, Luz M, Barclay L, Mohr E, Mooney L, Coulthard E, Lawrence A, Daniels S, Whone AL (2012) Assessing the benefit and safety of administering intermittent GDNF infusions in PD. Mov Disord 27(Suppl 1):S146

17. EU Clinical trials register https://www.clinicaltrialsregister.eu/ctrsearch/trial/2011-003866-34/GB

18. Luz M, Mohr E, Fibiger HC (2015) GDNF-induced cerebellar toxicity: a brief review. Neurotoxicology 52:46-56

19. Hadaczek P, Johnston L, Forsayeth J, Bankiewicz KS (2010) Pharmacokinetics and bioactivity of glial cell line-derived factor (GDNF) and neurturin (NTN) infused into the rat brain. Neuropharmacology 58:1114-1121

20. Taylor H, Barua N, Bienemann A, Wyatt M, Castrique E, Foster R, Luz M, Fibiger C, Mohr E, Gill S (2013) Clearance and toxicity of recombinant methionyl human glial cell line-derived neurotrophic factor( r-metHu GDNF) following acute convection-enhanced delivery into the striatum. PLoS One 8(3):e56186

21. Morrison PF, Laske DW, Bobo H, Oldfield EH, Dedrick RL (1994) High-flow microinfusion: tissue penetration and pharmacodynamics. Am J Phys 266:R292-R305
22. Kim JH, Mareci TH, Sarntinoranont M (2010) A voxelized model of direct infusion into the corpus callosum and hippocampus of the rat brain: model development and parameter analysis. Med Biol Eng Comput 48:203-214

23. Sarntinoranont M, Chen X, Zhao J, Mareci TH (2006) Computational model of interstitial transport in the spinal cord using diffusion tensor imaging. Ann Biomed Eng 34(8):1304-1321

24. Gimenez F, Krauze MT, Valles F, Hadaczek P, Bringas J, Sharma N, Forsayeth J, Bankiewicz KS (2011) Image-guided convection-enhanced delivery of GDNF protein into monkey putamen. NeuroImage 54:S189-S195

25. Begley DJ, Khan EU, Rollinson C, Abbott J (2000) The role of brain extracellular fluid production and efflux mechanisms in drug transport to the brain. In: Begley DJ, Bradbury MW, Kreuter J (eds) The blood-brain barrier and drug delivery to the CNS. Marcel Dekker, New York, pp 93-108

26. Zhan W, Kang GA, Glass GA, Zhang Y, Shirley C, Millin R, Possin KL, Nezamzadeh M, Weiner MW, Marks WJ, Schuff N (2012) Regional alterations of brain microstructure in Parkinson's disease using diffusion tensor imaging. Mov Disord 27(1):90-97

27. Sykova E, Nicholson C (2008) Diffusion in brain extracellular space. Physiol Rev 88:1277-1340

28. Wang P, Olbricht WL (2010) Retro-convection enhanced drug delivery: a computational study. Ann Biomed Eng 38(8):2512-2519

29. Stroh M, Zipfel WR, Williams RM, Webb WW, Saltzman WM (2003) Diffusion of nerve growth factor in rat striatum as determined by multiphoton microscopy. Biophys J 85(1):581-588

30. Wolak DJ, Thorne RG (2013) Diffusion of macromolecules in the brain: implications for drug delivery. Mol Pharm 10:1492-1504

31. Yin D, Valles FE, Fiandaca MS, Forsayeth J, Larson P, Starr P, Bankiewicz KS (2009) Striatal volume differences between nonhuman and human primates. J Neurosci Methods 176(2):200-205

32. Asthagiri AR, Walbridge S, Heiss JD, Lonser RR (2011) Effect of concentration on the accuracy of convective imaging distribution of a gadolinium-based surrogate tracer. J Neurosurg 115:467-473

33. Linninger AA, Somayaji MR, Mekarski M, Zhang L (2008) Prediction of convection-enhanced drug delivery to the human brain. J Theor Biol 250:125-138

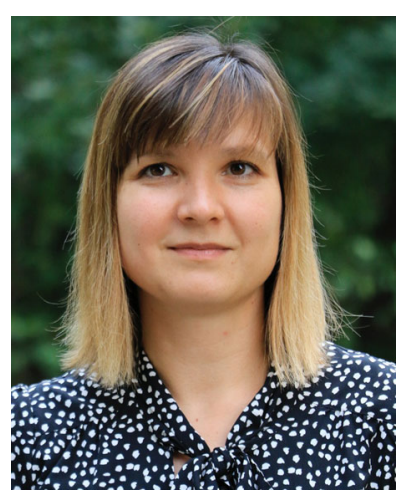

Dr. Elena Belova is a Senior Scientist in the Modeling and Simulation Group within the Department of Pharmacokinetics, Dynamics, and Metabolism at Pfizer, and her focus is a modeling support of the neuroscience/pain research programs. 


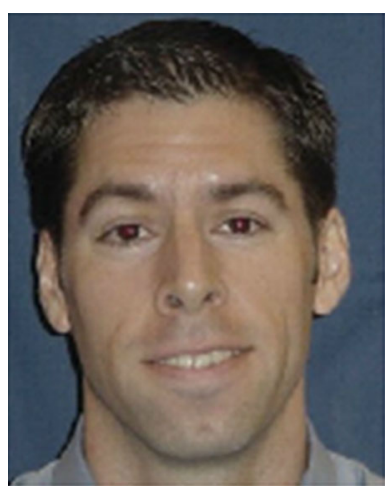

Dr. Christopher Shaffer holds a BS in chemistry (high honors) from The College of William \& Mary and a M.S. and Ph.D. (honors) in medicinal chemistry from the University of Kansas. Immediately following graduate school, Chris joined the Department of Pharmacokinetics, Dynamics, and Metabolism (PDM) as a biotransformation lab head working on CNS projects from preclinical development through POC. Four years later, Chris became a PDM lab head in CNS Discovery, where he worked for 9 years. In addition to PDM project representative roles on numerous CNS projects, Chris was the Research Project Leader for a number of programs. In August 2014, Chris formally joined the Neuroscience \& Pain Research Unit (NPRU) as a Portfolio Lead within the NeuroOpportunities Group. Chris has led many neuroscience-centric interdisciplinary efforts such as translational neuropharmacology and accurately projecting the human pharmacokinetics and neuropharmacokinetics of small molecules to optimize their clinical dosing regimens. Chris has co-authored 40 peer-reviewed manuscripts, speaks regularly in diverse external forums, and is a member of the American Chemical Society and its Medicinal Chemistry Division as well as the Society for Neuroscience.

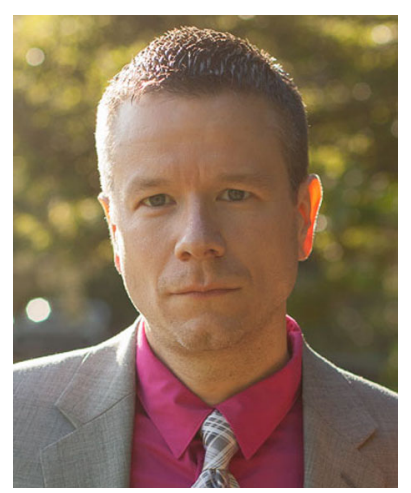

Dr. Patrick Trapa leads the neuroscience/pain quantitative translation group within the Department of Pharmacokinetics, Dynamics, and Metabolism at Pfizer. His team is primarily responsible for supporting projects from early discovery through candidate selection. Patrick received his bachelor's and Ph.D. from MIT in Materials Science and Engineering and an M.B.A. from the University of Rhode Island. After completing his doctoral work, he co-founded a lithiumpolymer-battery company and later served as lecturer and research associate at MIT. During this time, he also consulted with NASA on hightemperature metallurgy projects. He then ran the specialty battery division at a publicly traded battery company before entering Pfizer in 2006. Patrick has held roles in preformulations as well as ADME, PK/PD, and systems modeling. He presently leads several PBPK efforts in addition to actively supporting the neuroscience/pain portfolio. 